\title{
Performance Evaluation of MAC Protocols with Multi-Sink for Mobile UWSNs
}

\author{
Areeg Fahad Rasheed, A E Abdelkareem \\ College of Information Engineering Al-Nahrain University, Baghdad, Iraq. \\ E-mail: Areeg.Fahad@coie-nahrain.edu.iq
}

Received: 12 April 2019; Accepted: 19 May 2019; Published: 08 July 2019

\begin{abstract}
Currently, Underwater Wireless Sensor Network (UWSN) is one of modern science and have attached many researchers especially how interesting with Wireless Sensor Network (WSN). A mobile node in UWSN is one of the powerful mechanisms, it can be used for many aquatic applications such as Surveillance for wildlife, Pollution control, Military applications, etc. UWSN is different from the terrestrial network in terms of using acoustic waves as a communication medium. Due limitation of bandwidth, high latency, and long propagation delay of acoustic waves, many proper MAC protocols that work efficiently in Static UWSN are not suitable for the mobile network. In this article two MAC protocols appropriate for mobile underwater network Broadcast as Direct access and geo-routing aware MAC protocol (GOAL) as Handshaking are evaluated, in both static and mobile nodes with respect to throughput, energy consumption, and delay. Then the effect of using Multi-Sink on the performance is shown, the evaluation is done based on AquaSim simulator, Aqua3D Animator, and AWK programming language have been used to extract the results. As a result, the performance has been improved by using multi-Sink with two types of MAC address, and for all the metrics.
\end{abstract}

Index Terms-AquaSim, Aqua3d, Direct access, GOAL, Broadcast, Handshaking, UWSN, WSN.

\section{INTRODUCTION}

Mobile nodes in UWSNs are a few of the important and modern fields that have become of interest by many researchers in recent years for their multiple uses in many areas such as Monitoring, Disaster prediction, and Military purposes[1-3].

Underwater data transmission is different from terrestrial transmission by adopting it on acoustic waves. These waves face several challenges such as the high latency, narrow bandwidth, relatively limited energy sources $[1,3,4]$ as well as the movement in the environment that often causes a loss in the path and timevarying shifting, therefore many MAC protocols are unsuitable for underwater work when the nodes are in a state of mobility, so in this paper a comparison between better MAC protocols that work in mobile underwater network efficiently is produced, Broadcast as Direct access and GOAL as Handshaking. Moreover, we have improved the efficiency of the network using Multi-Sink network architecture.

Indirect access MAC protocols, sensor node does not need to know whether the channel is reserved or not by the other nodes. When they have ready packets, they will send directly to the channel, while in Handshaking approaches, each sensor node must exchange control packet Request-To-Send (RTS) and Clear-To-Send (CTS) in order to reserve the channel before transferring its data.

The aims of this article to improve the network efficiency by using suitable MAC protocols with the underwater mobile environment, also using multi-sink approaches for enhancement of packet delivery ratio with energy consumption for MAC protocol with mobile and static node.

This paper is organized into five sections. In section II, the most prominent work that has a relation to ours is discussed. In section III, the system model and analysis mechanism work of protocols are shown. In IV, performance evaluation of the Direct access and Handshaking protocols with Single Sink and Multi-Sink, and in section $\mathrm{V}$, the conclusion of the results are extracted.

\section{RELATED WORK}

Many research works have been done on an underwater MAC protocol in the last few years, which debated many challenges in detail $[1,2,3]$. Several MAC protocols also propose for different purposes including the reduction of energy consumption such as RMAC, which depending on three stages for prevention more than one sensor node on sending their data packets at the same time, and the UWANMac which making synchronize with each node to the same reason in RMAC [6,7]. That interested in increasing throughput such as (ALOHA and Slotted ALOHA) $[8,9]$ by alow each sensor to direct send data packet without sense the channel if it reserve by another node or not. They are concerned with minimizing collisions by control over the entire channel (FAMA and slotted FAMA) [10,11]. In addition, there are protocols that combine several features such as reducing energy consumption, take control of the channel, and increasing throughput such as (COPE and TMAC) $[12,13]$. However, these protocols, despite their 
efficiency, do not fit together in a mobile node environment. Thus, in this paper, an investigation and performance analysis of an appropriate underwater MAC protocol suitable for the mobile environment is produced with two underwater environment multi-sink and Single sink.

\section{SYSTEM MODEL}

\section{A. Network configuration}

Figure 1 illustrates our UWSN Architecture using Aqua3D animator [14] a Various number of nodes are utilized. These nodes are randomly distributed in the sea, gathering data and send it to the sink nodes which is positioned on the surface of the water. VBF is used as a routing protocol to forward data packets [14], and each node in a mobile node is integrated with an autonomous underwater vehicle (AUV), which is used to help nodes to move in underwater. It's powered and controlled by an operator/pilot via an umbilical or using remote control [15].

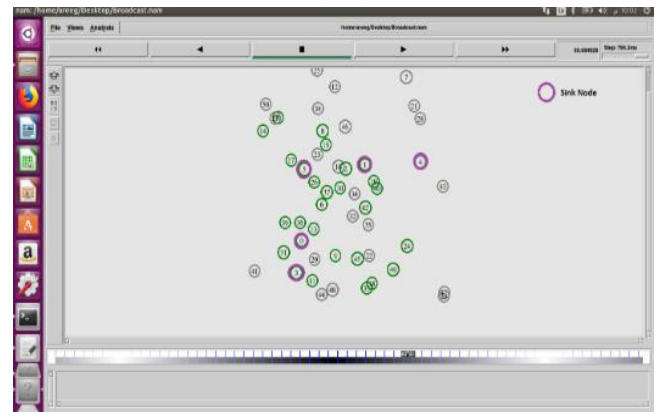

Fig.1. UWSN Architecture using NAM

\section{B. UNDERWATER MAC PROTOCOLS ANALYSIS}

\section{Broadcast MAC Protocols}

A simple Direct access underwater MAC protocol. The idea of Broadcast is when a sensor node is ready to send a packet, it must first check whether the channel is free or not, if the channel is free, the node duplicates the message and broadcast to all nodes in the network. Once the package is received, each node checks MAC and only the destination node receives the packet, while all others will neglect it. In the Broadcast protocol, the node does not need to send ACK for packets arrived. The algorithm 1 describes the operation of broadcast MAC protocol.

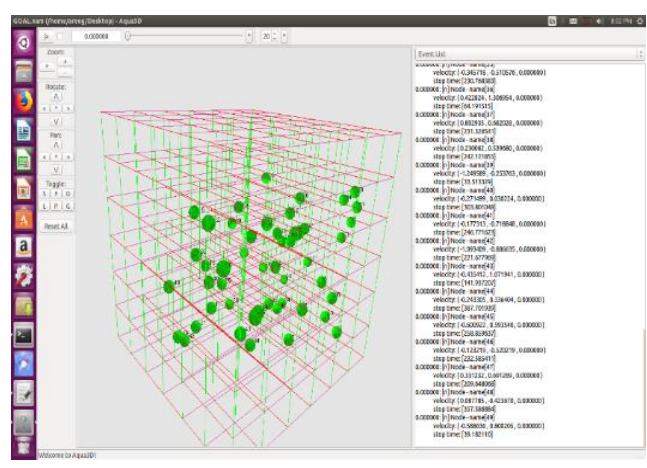

Fig.2. UWSN Architecture using Aqua3D

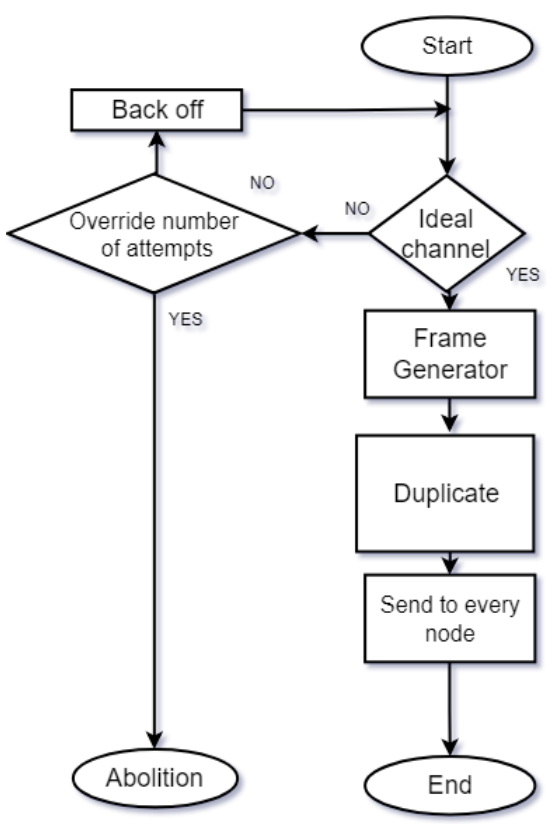

Fig.3. Broadcast Flowchart

\begin{tabular}{c}
\hline Algorithm 1: Broadcast Algorithm \\
\hline$i \leftarrow 0 ;$ \\
$i_{M A X} \rightarrow$ Maximum Iteration \\
while $i \neq i_{\text {MAX }}$ do \\
$\begin{array}{c}\text { Packet Generator } \\
\text { if } \text { channel free then } \\
\text { go to duplicated packet } \\
\text { Send its to Neighbors } \\
\text { else } \\
\text { go to BackOff Time } \\
\text { end } \\
\text { i+=1; } \\
\text { end }\end{array}$
\end{tabular}




\section{GOAL MAC Protocols}

(Geo-routing aware mac protocol) [16] proposed for underwater to data transmission. It integrates three mechanisms, self-adaptation algorithm based RTC/CTS handshake, for selecting the better forward node and reservation, Cyber Carrier Sense, for reducing collision.

Among data packet and implicit ACK packet, for improving end to end reliability. To simplify the selfadaptation algorithm based on RTS/CTS handshake, we will take network topology in Fig.3. As an example, when current forwarder $\mathrm{F}$ has data ready for transfer, it broadcasts RTS packet which contains sender, receiver, forwarder location, and Time of the future data transmission (T). After receiving RTS, each neighbor applies self-adaption algorithm that use in geo routing protocol such as (VBF, HHVBF, VBVA), then neighbor with less distance to the forwarder node will respond CTS packet which contains its location and relative time ( $\mathrm{T}^{\prime}$ it will send data packet to node $\mathrm{F}$ as shown in Fig.4. After that, node $\mathrm{F}$ waits for a random time to receive CTS if not, it tries to broadcast RTS again, otherwise, it selects next hope by applied self - adaptation again, ultimately the node F starts sending data. According to the selfadaptation algorithm, due to node $B$ have a minimum distance to the destination than other neighbors of $\mathrm{F}$, it will be selected as the best next hope for the forward data packet.

In Cyber Carrier Sense mechanism when node B receives the RTS packet as shown in Fig.4. It will be known to receive data packet during the time.

$$
[T-T R T S, T-T R T S+T D A T A]
$$

From F, where TRTS is transmission time of RTS. In order to avoid a collision, it must not deliver packets during

$$
\left[T^{\prime}-\text { TCTS } 2 \text { - Tprop }, T^{\prime}-\text { TCTS - 2Tprop + TDATA }\right]
$$

As a result of the mobile nodes being in the underwater environment the guard time use for tolerating error, in this case, the propagation delay is in the range [Propagation - $\mathrm{T}$ guard, Propagation guard]. Therefore, the avoid period becomes

$$
\begin{gathered}
{\left[T^{\prime}-\text { TCTS }-2 T \text { prop }-2 T \text { guard }^{\prime}-T C T S-\right.} \\
2 T \text { peop }+ \text { T DAT A }+2 T \text { g guard }]
\end{gathered}
$$

While in Implicit acknowledgment mechanism. For more reliability, the node waits to receive ACK to ensure that the packet arrives Successfully. Otherwise, it retransmits RTC/CTS/DATA again. The maximum number of retransmissions should be specific to avoid more delay and energy consumption.

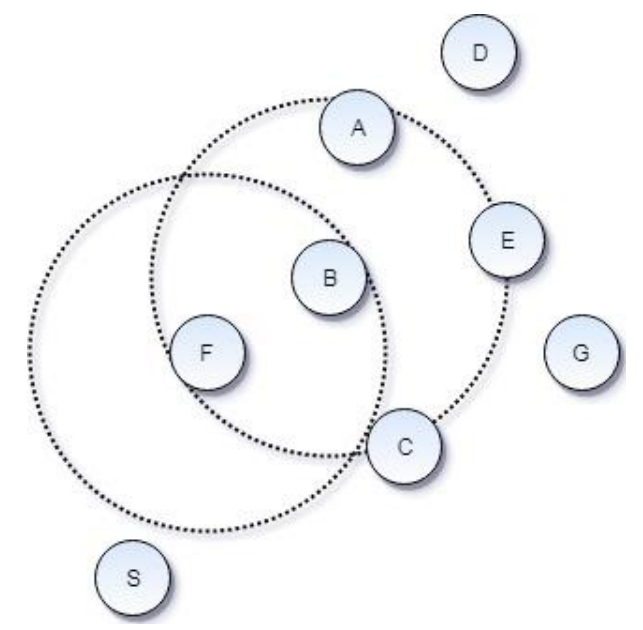

Fig.4. GOAL Example

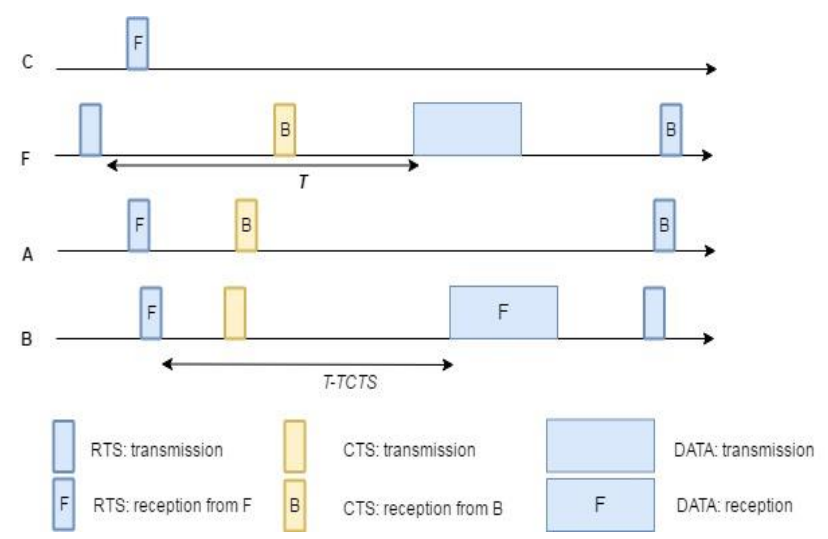

Fig.5. GOAL Data Transmission

\section{PERformance Evaluation}

In this section, performance evaluation of the Broadcast and Goal underwater MAC protocols is produced in terms of Energy consumption, Receive throughput , and Average End to End delay in both static and mobile nodes and by using single-sink and multi-sink approaches, system parameters are used for the first and second scenario as shown in Table 1 and Table 2 respectively. Aqua-Sim and Aqua3D are used as network simulation and Animator respectively. Aqua-Sim designed by Zheng Peng in University of Connecticut [17] 
for underwater sensor networks, it was developed basis on NS-2 and can use effectively to simulate acoustic signal attenuation and packet collisions in UWSNs. Aqua-Sim work in parallel and independent with Carnegie Mellon University (CMU) wireless simulation package. Aqua-Sim simulator is organized into three folders, uwmac, uwrouting, and uwcommon. MAC protocols and the codes simulating acoustic channels grouped in the folder of uwmac. Routing protocol grouped in uwrouting while the traffic and codes simulating underwater sensor nodes are organized in a uwcommon folder, also Aquasim used Thorp's model as channel model. Fig.5. Shows the architecture of AquaSim simulator. [17].

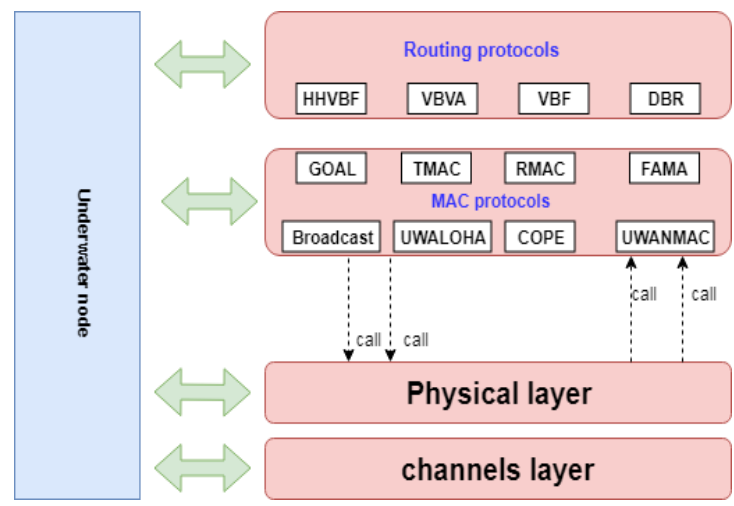

Fig.6. Aqua-Sim simulator architecture
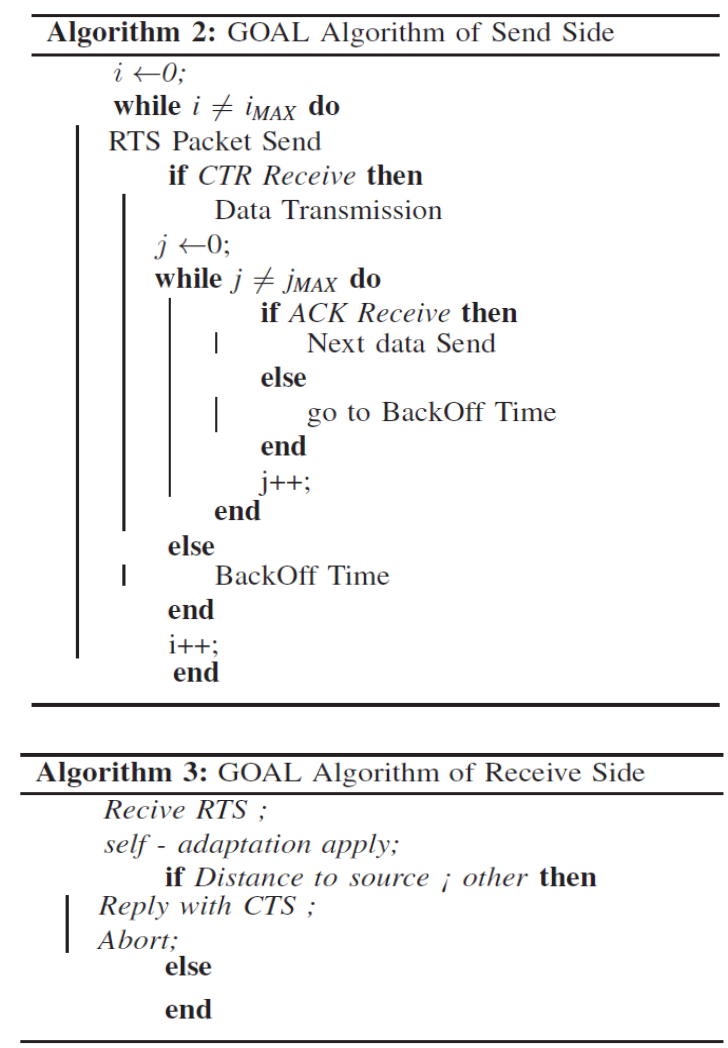

Table 1. System Parameters of Mobile Node.

\begin{tabular}{|c|c|}
\hline Parameters & Value \\
\hline Simulation Time & $1000 \mathrm{sec}$ \\
\hline Area & $1000 \mathrm{~m} * 1000 \mathrm{~m} * 500 \mathrm{~m}$ \\
\hline Number of Nodes & Varied \\
\hline Routing Protocols & Vector base forward \\
\hline Mac protocol & BroadcastMac GOAL \\
\hline Data Rate & $20 \mathrm{Kpbs}$ \\
\hline Packet size & $60 \mathrm{byte}$ \\
\hline Transmission Power & $1 \mathrm{~W}$ \\
\hline Maximum Speed & $5 \mathrm{~m} / \mathrm{s}$ \\
\hline Minimum speed & $0.5 \mathrm{~m} / \mathrm{s}$ \\
\hline Antenna & OmniAntenna \\
\hline Propagation model & Underwater Propagation \\
\hline
\end{tabular}

Table 2. System Parameters of Static Node

\begin{tabular}{|c|c|}
\hline \multicolumn{1}{|c|}{ Parameters } & $\begin{array}{c}\text { Value } \\
\text { Simulation Time }\end{array}$ \\
\hline Area & $4000 \mathrm{sec}$ \\
\hline Number of Nodes & $\mathbf{2 6}$ \\
\hline Routing Protocols & Vector base forward \\
\hline Mac protocol & Broadcast, GOAL \\
\hline Data Rate & $20 \mathrm{Kpbs}$ \\
\hline Packet size & $60 \mathrm{byte}$ \\
\hline Transmission Power & $2 \mathrm{~W}$ \\
\hline Antenna & Omni Antenna \\
\hline Propagation model & Underwater Propagation \\
\hline No. of Sink Node & 5 \\
\hline
\end{tabular}

As shown in Fig.8. the Receive throughput of GOAL MAC protocol is more efficient than the Broadcast because the GOAL MAC is using the RTS/CTS for channel reservation, thus reduces the packet collision and the ACK that is used to improve the reliability of end-toend reception. In addition to that when using Multi-sink approaches is improved the efficiency of the network for both the GOAL and Broadcast. The reason behind that is when using the multi-sink, the load on the channel is reduced so that it can decrease the number of packets collision where it becomes a more than one option to send data.

From Fig.9. the GOAL mac protocols are more energy efficient than the Broadcast due to it using the selfadaptation simultaneously with RTS/CTS handshake so this mechanism allows the node to dynamically find out the better next-hop with low route discovery cost. Moreover, using a multi-sink approach improves the energy consumption as we mentioned earlier which can reduce the load on channels thus reducing the number of packet collision and as a result, fewer packets need to be retransmitted. Where pregnancy on the nodes becomes less compared to pregnancy when only one sink is used. Fig.10. clarifies that Broadcast is more efficient in case of average End-to-End delay than the GOAL mac protocols 
due to the best-effort protocol, which attempts to transmit packets to their purposed destinations but that does not provide any special features for retransmitting corrupted or lost packets. While the GOAL MAC is based on RTS/CTS schema for reservation channels before DATA transmission and implicit acknowledgment that often consume more time. Use multi-sink can reduce almost in half the average end-to-end time for both the broadcast and GOAL protocols.

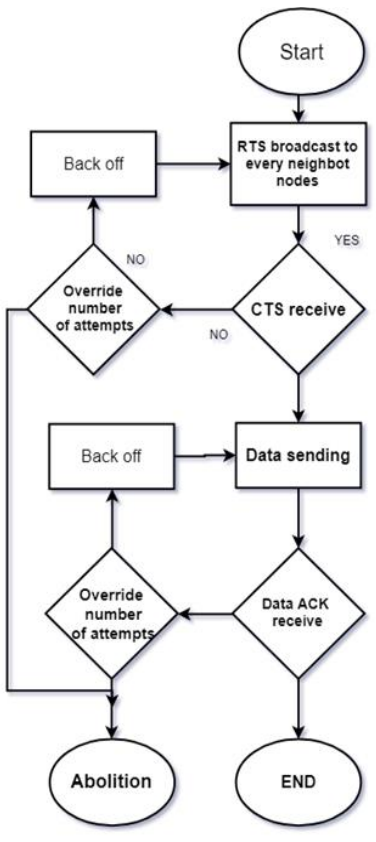

(a) Sender Side

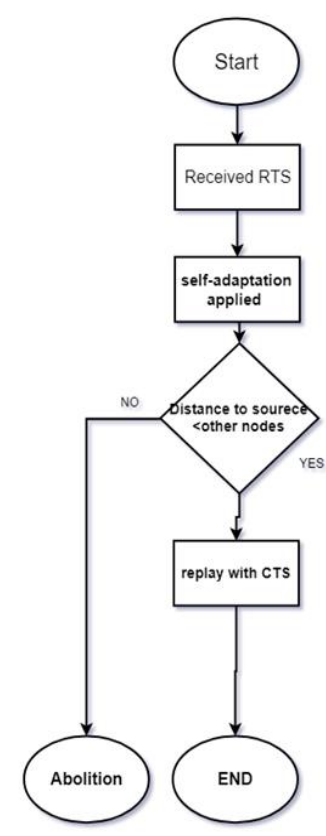

(b) Receive Side

Fig.7. GOAL Flowchart

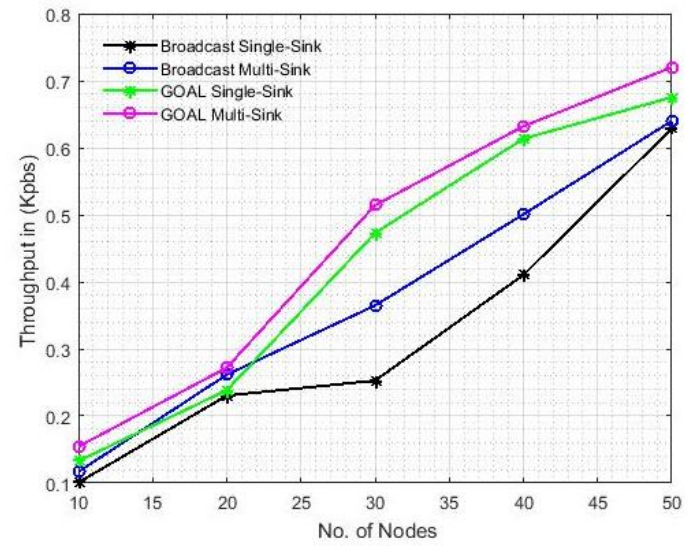

Fig.8. Receive throughput in (Kpbs).

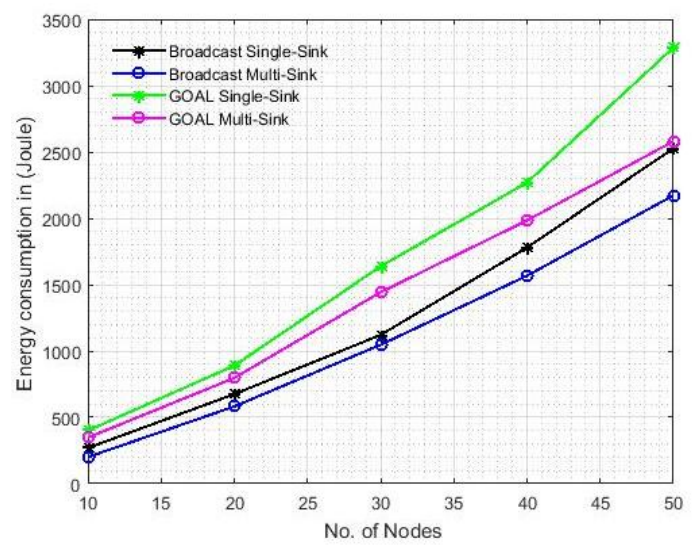

Fig.9. Energy consumption in (Joule).

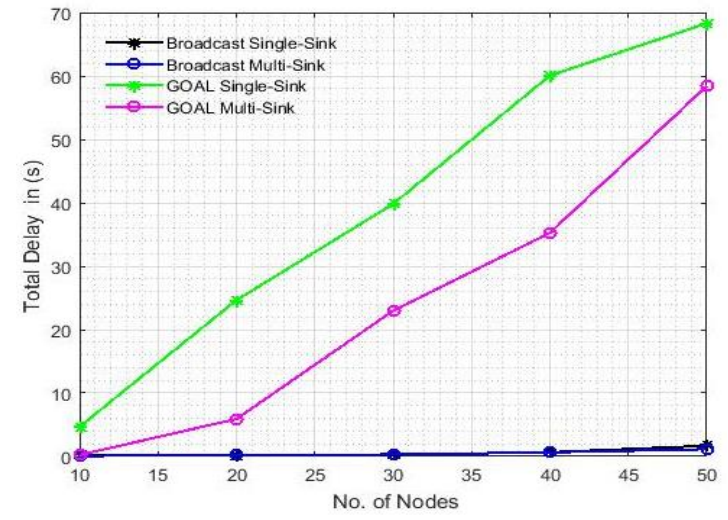

Fig.10. Total Delay in s.

Fig.11. shows the performance of Broadcast MAC with static node and when the number of nodes exceeds 25 it is more efficient than GOAL mac protocols with respect to receiving throughput, because in GOAL when the number of nodes is large, there is an increased exchange of control packets thus, more collisions between packets. This situation appears less with GOAL MAC in the case of the static node because the node changes the location and the path of sending packets permanently, resulting in a collision between the nodes less than the static state. Fig.12. The energy consumption in both scenarios is almost constant. In both cases, the consumption of the Broadcast is more than GOAL because it is simple to rely on direct transmission, unlike the GOAL protocol, Which depends on competition between the contract 
before transmission. This effect plays the same role in both scenarios. The same thing applies in relation to Delay as shown in Fig.13. In addition to that using multisink approaches increased transmission options for each node and this assist to improve the network efficiency almost to all metris (receive throughput, energy consumption, and delay) as shown in Table 3. Table4 presents the findings result from Aquasm Simulation.

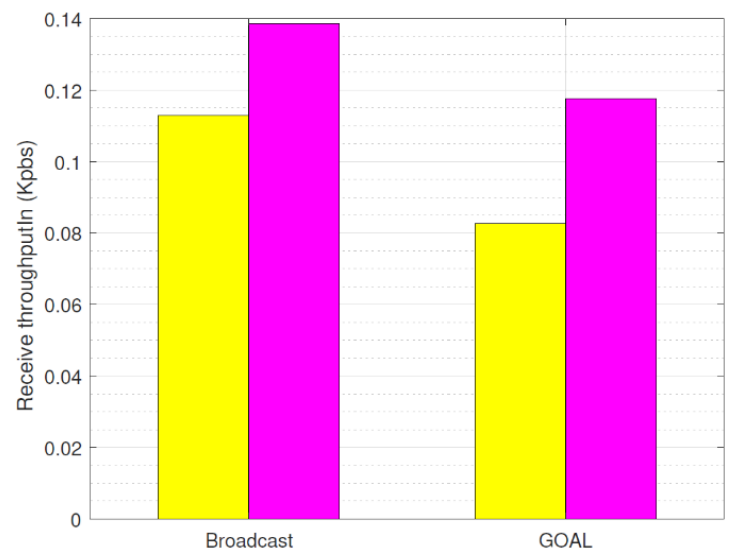

Fig.11. Receive Throughput in (Kbps).

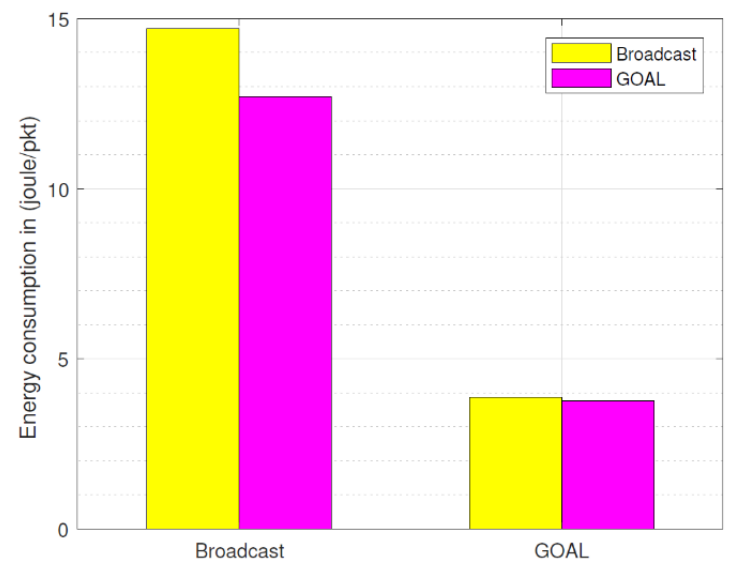

Fig.12. Energy consumption in (joule/pkt)

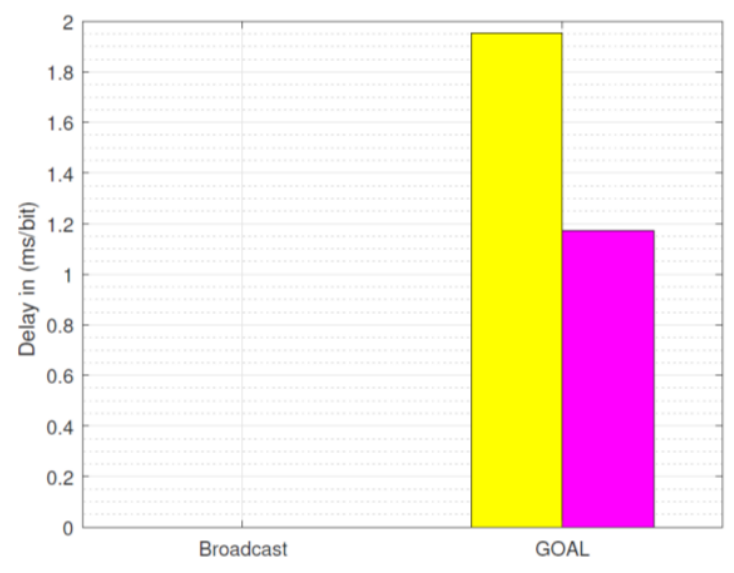

Fig.13. Delay in (ms/bit).
Table 3. Simulation result of UWSN mobile MAC-protocols.

\begin{tabular}{|c|c|c|c|c|c|}
\hline \multicolumn{6}{|c|}{ Received throughput in (Kpbs) } \\
\hline \multicolumn{6}{|c|}{ Single Sink } \\
\hline No of node & 10 & 20 & 30 & 40 & 50 \\
\hline Broadcast & 0.1008 & 0.2304 & 0.2528 & 0.4104 & 0.6288 \\
\hline GOAL & 0.1328 & 0.2384 & 0.4728 & 0.6136 & 0.6752 \\
\hline \multicolumn{6}{|c|}{ Multi Sink } \\
\hline No of node & 10 & 20 & 30 & 40 & 50 \\
\hline Broadcast & 0.1168 & 0.2616 & 0.3648 & 0.5008 & 0.64 \\
\hline GOAL & 0.1544 & 0.272 & 0.5144 & 0.632 & 0.72 \\
\hline \multicolumn{6}{|c|}{ Energy consumption in (Joule) } \\
\hline \multicolumn{6}{|c|}{ Single Sink } \\
\hline No of node & 10 & 20 & 30 & 40 & 50 \\
\hline Broadcast & 269 & 675 & 1121 & 1779 & 2527 \\
\hline GOAL & 200 & 582 & 1047 & 1567 & 2172 \\
\hline \multicolumn{6}{|c|}{ Multi Sink } \\
\hline No of node & 10 & 20 & 30 & 40 & 50 \\
\hline Broadcast & 400 & 890 & 1638 & 2270 & 3286 \\
\hline GOAL & 347 & 797 & 1443 & 1984 & 2580 \\
\hline \multicolumn{6}{|c|}{ Total Delay in ms } \\
\hline \multicolumn{6}{|c|}{ Single Sink } \\
\hline No of node & 10 & 20 & 30 & 40 & 50 \\
\hline Broadcast & 280 & 94 & 286 & 653 & 1684 \\
\hline GOAL & 4691 & 24631 & 39817 & 60066 & 68295 \\
\hline \multicolumn{6}{|c|}{ Multi Sink } \\
\hline No of node & 10 & 20 & 30 & 40 & 50 \\
\hline Broadcast & 69 & 280 & 308 & 645 & 1152 \\
\hline GOAL & 226 & 5910 & 22974 & 35262 & 58410 \\
\hline
\end{tabular}

Table 4. Increase percentage by using Multi-Sink for Static and Mobile

\begin{tabular}{|c|c|c|c|}
\hline \multicolumn{4}{|c|}{ Mobile Node } \\
\hline Protocols & Throughput & Energy & Delay \\
\hline Broadcast & $20 \%$ & $13 \%$ & $95 \%$ \\
\hline GOAL & $30 \%$ & $2 \%$ & $38 \%$ \\
\hline \multicolumn{4}{|c|}{ Static Node } \\
\hline Protocols & Throughput & Energy & Delay \\
\hline Broadcast & $8 \%$ & $21 \%$ & $40 \%$ \\
\hline GOAL & $30 \%$ & $2 \%$ & $38 \%$ \\
\hline
\end{tabular}

\section{CONCLUSION}

In this paper, performance analysis and comparison of the Direct access and Handshaking Mac protocols that are suitable to work with static and mobile node (Broadcast and GOAL) with respect to Energy consumption, Receive throughput and Average E2E delay. Two types of network architecture are considered, which are MultiSink and Single-Sink. This paper shows that GOAL MAC protocol is more efficient in Energy consumption and Receive throughput than Broadcast for the moving network architecture while the Broadcast is more efficient in Receive throughput than GOAL in Static network. Therefore, it is preferable to use the Broadcast in realtime applications with static networks because there are 
less delay and high throughput than GOAL. While for long time mobile applications, GOAL is more appropriate because it consumes less energy, and this can help the network for longer period work. Moreover, by using Multi-Sink approach the network efficient overall improved to more than half for both of GOAL and Broadcast.

\section{REFERENCES}

[1] J. Heidemann, M. Stojanovic, and M. Zorzi, "Underwater sensor networks: applications, advances, and challenges," Phil. Trans. R. Soc. A vol. 370, no. 1958, pp. 158-175, 2012.

[2] E. Felemban, F. K. Shaikh, U. M. Qureshi, A. A. Sheikh, and S. B. Qaisar, "Underwater sensor network applications: A comprehensive survey," International Journal of Distributed Sensor Networks, vol. 11, no. 11, p. 896832, 2015.

[3] J. Heidemann, W. Ye, J. Wills, A. Syed, and Y. Li, "Research challenges and applications for underwater sensor networking," Wireless Communications and Networking Conference, 2006. WCNC 2006. IEEE, vol. 1. IEEE, 2006, pp. 228-235.

[4] F. Akyildiz, D. Pompili, and T. Melodia, "Underwater acoustic sensor networks: research challenges" Ad hoc networks, vol. 3, no. 3, pp. 257-279, 2005.

[5] E. M. Sozer, M. Stojanovic, and J. G. Proakis.,"Underwater acoustic networks," IEEE journal of oceanic engineering, vol. 25, no. 1, pp. 72-83, 2000.

[6] P. Xie and J.-H. Cui, " R-mac: An energy-efficient mac protocol for underwater sensor networks," International Conference on Wireless Algorithms, Systems and Applications. IEEE, 2007, pp. 187-198.

[7] M. K. Park and V. Rodoplu, "Uwan-mac: An energyefficient mac protocol for underwater acoustic wireless sensor networks," IEEE journal of oceanic engineering, vol. 32, no. 3, pp. 710-720, 2007.

[8] J. K. Parmar and M. Mehta, "Performance evaluation of broadcast mac and aloha mac protocol for underwater wireless sensor networks".

[9] P. Mandal, S. De, and S. S. Chakraborty, "A receiver synchronized slotted aloha for underwater wireless networks with imprecise propagation delay information," Ad Hoc Networks, vol. 11, no. 4, pp. 1443-1455, 2013.

[10] C. L. Fullmer and J. Garcia-Luna-Aceves,"Floor acquisition of multiple access (fame) for packet-radio networks. ACM SIGCOMM computer communication

[11] M. Molins and M. Stojanovic, "Slotted fama: a mac protocol for underwater acoustic networks," OCEANS 2006-Asia Pacific. IEEE, 2007, pp. 1-7.

[12] Z. Peng, Y. Zhu, Z. Zhou, Z. Guo, and J.-H. Cui," Copemac: A contention-based medium access control protocol with a parallel reservation for underwater acoustic networks," OCEANS 2010 IEEE-Sydney,pp. 1-10.

[13] R. Yadav, S. Varma, N. Malaviya et al, "A survey of mac protocols for wireless sensor networks," UbiCC Journal, vol. 4, no. 3, pp. 827-833, 2009.

[14] M. Tran, M. Zuba, S. Le, Y. Zhu, Z. Peng, and J.-H. Cui, "Aqua- 3d: An underwater network animator," Oceans, IEEE, 2012, pp. 1-5.

[15] L. Paull, S. Saeedi, M. Seto, and H. Li.AUV navigation and localization: A review. In IEEE Journal of Oceanic Engineering, vol. 39, no. 1, pp. 131-149, 2014.

[16] Y. Zhu, R. Z. Zhou, J. P. Zheng, and J.-H. Cui. An efficient georouting aware mac protocol for underwater acoustic networks. In International Conference on Ad Hoc
Networks. Springer, 2010, pp. 185-200.

[17] P. Xie, Z. Zhou, Z. Peng, H. Yan, T. Hu, J.-H. Cui, Z. Shi, Y. Fei, and S. Zhou," Aqua-sim: An ns-2 based simulator for underwater sensor networks," OCEANS 2009, MTS/IEEE Biloxi-marine technology for our future: global and local challenges IEEE, 2009, pp. 1-7.

[18] I. S. Institute. Ns-2. [Online]. Available: https://www.isi.edu/nsnam/ns/

[19] U. U. of Connecticut. Uwsn. [Online]. Available: http://obinet.engr.uconn.edu/wiki/index.php/Aqua-Sim

How to cite this paper: Areeg Fahad Rasheed, A E Abdelkareem, "Performance Evaluation of MAC Protocols with Multi-Sink for Mobile UWSNs", International Journal of Computer Network and Information Security(IJCNIS), Vol.11, No.7, pp.1-7, 2019.DOI: 10.5815/ijcnis.2019.07.01 